\title{
A multi-core fiber to single-mode fiber side-polished coupler
}

\author{
H. Zhang, ${ }^{1}$ N. Healy, ${ }^{1,2, *}$ H.C. Mulvad, ${ }^{1}$ J.R. Hayes, ${ }^{1}$ M.N. Petrovich, ${ }^{1}$ S. Dasgupta, ${ }^{1,3}$ D.J. Richardson, ${ }^{1}$ and \\ A.C. Peacock ${ }^{1}$ \\ ${ }^{I}$ Optoelectronics Research Centre, University of Southampton, Highfield, Southampton SO17 1BJ, UK \\ ${ }^{2}$ Emerging Technology and Materials Group, School of Electrical and Electronic Engineering, \\ Newcastle University, Newcastle upon Tyne NE1 7RU, UK \\ ${ }^{3}$ Lightcue, Bangalore, India \\ noel.healy@ncl.ac.uk
}

\begin{abstract}
Evanescent coupling between a side-polished multi-core fiber and a single-mode fiber is demonstrated for the first time. The low-loss tap coupler is capable of isolating the output from a single core of a 7-core fiber.

OCIS codes: (060.1810) Buffers, couplers, routers, switches, and multiplexers; (060.2310) Fiber optics
\end{abstract}

\section{Introduction}

Having been first demonstrated in 1979, multi-core fibers (MCFs) are currently undergoing a renaissance due to heightened interest in space-division multiplexing technologies. This surge in activity is fueled by the need to increase the capacity of the networks that modern day life has come to depend on [1]. Though complementary research has seen the realization of some important MCF devices, for example, multi-core erbium doped fiber amplifiers [2], some key fiber devices remain to be developed. One of the most important of these devices is the passive optical fiber coupler, which is used for splitting and tapping light, making it a ubiquitous component of current networks. In this paper, we report the first MCF to single-mode fiber coupler, which can tap light from a single core of a 7 -core fiber into that of a standard fiber.

Today, the most common type of optical fiber coupler is fabricated using the fused tapering method, a technique that requires the two fibers to be held in contact whilst being drawn in a heat-source. As a result, the fibers reduce in diameter and the mode in each fiber expands until light can resonantly couple between them. However, as with a number of fiber types, the refractive index profile of the MCF precludes tapering as a viable coupler fabrication technology, in this case, due to unwanted cross-talk between the MCF cores. Consequently, we have revisited the side-polishing approach to coupler fabrication. Side-polishing has been widely studied for making fiber-optic devices as it permits lateral access to the light in an optical fiber via interaction with the propagating mode's evanescent field. Here, we report a, low-loss, wide-band, side-polished tap coupler that can be used for signal monitoring in MCF networks.

\section{MCF coupler fabrication}

The MCF used in this work has an outer diameter of $198 \mu \mathrm{m}$ and was designed for single mode operation in the telecommunications region. It has seven $8 \mu \mathrm{m}$ diameter cores that are arranged in a hexagonal array with $50 \mu \mathrm{m}$ core-to-core pitch. The single-mode fiber is standard SMF-28 which has an $8 \mu \mathrm{m}$ core and a $125 \mu \mathrm{m}$ outer diameter. Both fibers were mounted in separate polishing blocks with special care taken to ensure that the polishing plane of the MCF fiber allowed access to just one core. Each polishing block was designed to produce a polished fiber with a $1 \mathrm{~cm}$ long uniform interaction region and adiabatic transitions from this region to the full-fiber so as to suppress transmission losses.

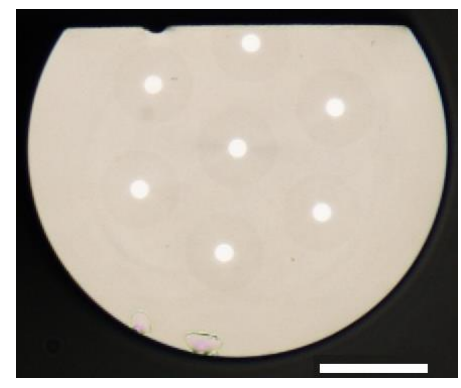

(a)

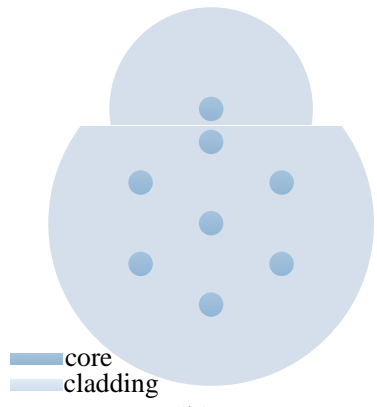

(b)

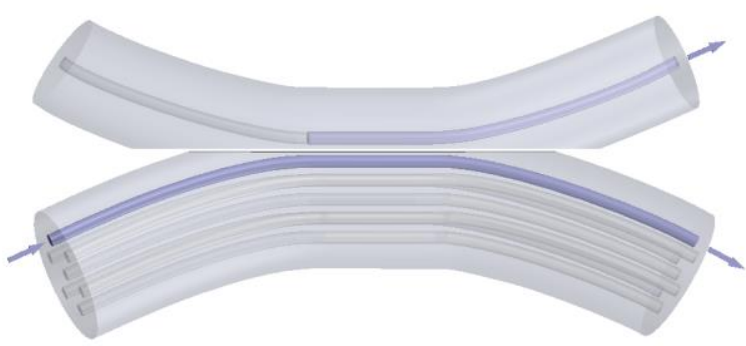

(c)

Fig. 1. (a) Microscope image of the cross-section of a side-polished MCF. Scale bar: $50 \mu \mathrm{m}$. A dip on the polished surface is due to a key hole in the MCF. (b) Schematic of the cross-section at the coupler's interaction region. (c) Illustration of the coupler showing light coupling from the MCF to the single-mode fiber. Arrow: light input and output. 
A portion of the cladding was then removed from each fiber using a standard polishing technique. Polishing was terminated when the polished surface reached a distance of approximately $1 \mu \mathrm{m}$ from the target core. Fig. 1(a) shows the cross-section of a polished MCF fiber and proves low polished surface roughness to keep it low-loss. The two polished blocks were then brought together and aligned so that evanescent coupling between the cores was observed. Fig. 1(b) shows a schematic image of the cross-section of the coupler illustrating the geometry and orientation of the polished fibers and Fig. 1(c) illustrates the coupling of light from one core of the MCF into the single-mode fiber.

\section{Characterization and discussion}

Optimization and tuning of the coupling was achieved by mounting one of the polished blocks in a fixed assembly and mounting the second block in an assembly with a kinematic mount with sub-micron positioning accuracy. The latter block was adjusted to increase or decrease the coupling efficiency by changing the gap distance between the two blocks. A matching liquid with index equal to the fiber cladding was applied in the gap. The spectral response of the device was observed using an ASE source in conjunction with an optical spectrum analyzer. Fig. 2 shows the spectra at the single-mode fiber output port with different tuning settings. The coupler could be optimized so that the coupling into the single-mode fiber tap was flat at $\sim 0.35 \%$ and varied to more than $2.0 \%$ within a $0.5 \mu \mathrm{m}$ distance change. The wide transmission window is from $1535 \mathrm{~nm}$ to $1615 \mathrm{~nm}$, including the entire telecommunications Cband. The loss of the coupler was measured to be $0.4 \mathrm{~dB}$ at an operation wavelength of $1550 \mathrm{~nm}$, a value that could be improved with further optimization on the experiment setup.

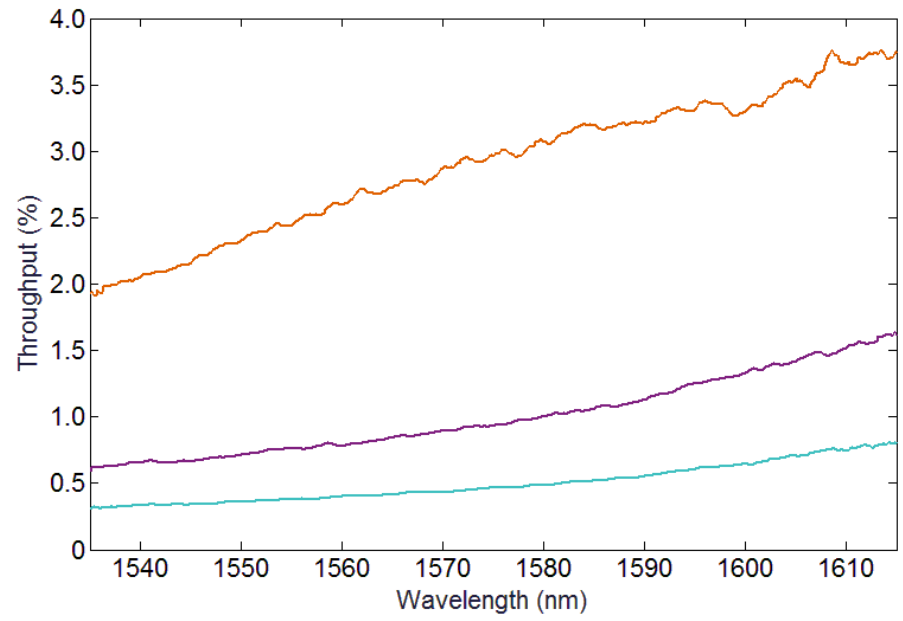

Fig. 2 Spectral response of the coupler for a number of tunings. The gap between the two fibers was varied over $0.5 \mu \mathrm{m}$ range.

\section{Conclusion}

Using a modified side-polishing technique, we have produced the first demonstration of evanescent field coupling between an MCF and a single-mode fiber. Furthermore, we have demonstrated a level of tunability and a flat wideband spectral response. It is anticipated that the polishing depth and interaction length will give further control over the coupling characteristics, such as spectral flatness and coupling ration. We expect such devices will form a critical component of future MCF networks, providing lateral access to the propagating light for monitoring, splitting and multiplexing applications.

This work was funded in part by EU projects COSIGN (grant number 61592) and SAFARI (grant number 642928).

\section{References}

[1] D. J. Richardson, J. M. Fini, and L. E. Nelson, “Space-division multiplexing in optical fibres,” Nat. Photon. 7, 354-362 (2013).

[2] K. S. Abedin, T. F. Taunay, M. Fishteyn, M. F. Yan, B. Zhu, J. M. Fini, E. M. Monberg, F. V. Dimarcello, and P. W. Wisk, "Amplification and noise properties of an erbium-doped multicore fiber amplifier," Opt. Express 19, 16715-16721 (2011). 